\title{
Bohring-Opitz (Oberklaid-Danks) syndrome: clinical study, review of the literature, and discussion of possible pathogenesis
}

\author{
Rob Hastings ${ }^{1}$, Jan-Maarten Cobben ${ }^{2}$, Gabriele Gillessen-Kaesbach ${ }^{3}$, Judith Goodship ${ }^{4}$, Hanne Hove ${ }^{5}$, \\ Susanne Kjaergaard ${ }^{5}$, Helena Kemp ${ }^{6}$, Helen Kingston ${ }^{7}$, Peter Lunt ${ }^{1}$, Sahar Mansour ${ }^{8}$, Ruth McGowan ${ }^{9}$, \\ Kay Metcalfe ${ }^{7}$, Catherine Murdoch-Davis ${ }^{6}$, Mary Ray ${ }^{10}$, Marlène Rio ${ }^{11}$, Sarah Smithson ${ }^{1}$, John Tolmie ${ }^{9}$, \\ Peter Turnpenny ${ }^{12}$, Bregje van Bon ${ }^{13}$, Dagmar Wieczorek ${ }^{14}$ and Ruth Newbury-Ecob ${ }^{\star, 1}$
}

Bohring-Opitz syndrome (BOS) is a rare congenital disorder of unknown etiology diagnosed on the basis of distinctive clinical features. We suggest diagnostic criteria for this condition, describe ten previously unreported patients, and update the natural history of four previously reported patients. This is the largest series reported to date, providing a unique opportunity to document the key clinical features and course through childhood. Investigations undertaken to try and elucidate the underlying pathogenesis of BOS using array comparative genomic hybridization and tandem mass spectrometry of cholesterol precursors did not show any pathogenic changes responsible.

European Journal of Human Genetics (2011) 19, 513-519; doi:10.1038/ejhg.2010.234; published online 2 February 2011

Keywords: Bohring-Opitz; Oberklaid-Danks; cholesterol biosynthesis; array CGH; trigonocephaly

\section{INTRODUCTION}

Bohring-Opitz syndrome (BOS) was first described in 1999 by Bohring et al, ${ }^{1}$ who described four new patients and identified similarities with two patients who had previously been reported as having Opitz C syndrome. ${ }^{2,3}$ As one of the patients was initially described by Oberklaid and Danks, ${ }^{2}$ this syndrome is sometimes known as Oberklaid-Danks syndrome, but we will use the more commonly used attribution of BOS henceforth. To our knowledge, a total of 21 cases have been reported in the medical literature to date. We propose diagnostic criteria for the clinical diagnosis of BOS, describe 10 previously unreported patients, and provide an update on four of those reported previously. ${ }^{4-6}$ The characteristic features of this condition are typical facial appearance (trigonocephaly/prominent metopic ridge, retrognathia, prominent eyes with hypoplastic supraorbital ridges, upslanting palpebral fissures, depressed nasal bridge, anteverted nares, low-set and posteriorly rotated ears, palatal abnormalities and broad alveolar ridges, flammeus nevus, low anterior hairline), microcephaly, IUGR and short stature, joint abnormalities, abnormal tone, severe/profound developmental delay, susceptibility to infections, feeding difficulties, and high infant mortality. Owing to the overlap of many of the clinical features of BOS with some of the disorders of cholesterol biosynthesis, such as Smith-Lemli-Opitz syndrome, desmosterolosis, and lathosterolosis, we decided to analyze cholesterol biosynthesis precursors using tandem mass spectrometry.

\section{PATIENTS AND METHODS}

Patients

In this study, 14 patients with the clinical features of BOS have been included. All patients had a normal karyotype on standard, G-banded, chromosome analysis.

As molecular confirmation of the diagnosis is not yet possible and there are no set diagnostic criteria, we have included those patients who have at least seven of the ten more common and/or specific features. These are: (1) trigonocephaly; (2) primary or secondary microcephaly; (3) flammeus nevus; (4) prominent eyes; (5) micro- or retrognathia; (6) abnormal palate; (7) typical 'BOS posture' (BOS posture of the upper limbs is defined as having three out of four features: exorotation and/or adduction of the shoulders; flexion at the elbows; flexion at the wrists; and ulnar deviation of the wrists and/or fingers at the MCP joints.); (8) feeding difficulties; (9) IUGR; and (10) severe/profound learning difficulties or death before this can be adequately assessed.

Biochemical study: analysis of the cholesterol biosynthesis pathway Cholesterol precursors were analyzed as described by Kelley. ${ }^{7}$ The precursors were liberated from their fatty acid esters by saponification using methanolic tetramethyl-ammonium hydroxide and extracted into a hydrophobic solvent. Extracts were then dried under nitrogen and converted to heat stable trimethylsilyl derivatives using trimethylchorosilane. Analysis was then performed by gas chromatography mass spectrometry, which both separated and detected the following cholesterol precursors: 7-dehydrocholesterol, 8-dehydrocholesterol, cholestanol, desmosterol, lathosterol, and lanosterol. Individual

${ }^{1}$ Clinical Genetics Department, Univerity Hospitals NHS Foundation Trust, St Michael's Hospital, Bristol, UK; ${ }^{2}$ Department of Pediatric Genetics, Emma Kinderziekenhuis AMC, Amsterdam, The Netherlands; ${ }^{3}$ Institut für Humangenetik, Universität zu Lübeck, Lübeck, Germany; ${ }^{4}$ Institute of Human Genetics, Newcastle University, Newcastle Upon Tyne, UK; ${ }^{5}$ Department of Clinical Genetics, University Hospital, Rigshospitalet, Copenhagen, Denmark; ${ }^{6}$ Department of Biochemistry, Southmead Hospital, Bristol, UK; ${ }^{7}$ Department of Genetic Medicine, MAHSC, St Mary's Hospital, Manchester, UK; ${ }^{3}$ Southwest Thames Regional Genetics Service, St George's Hospital, London, UK; ${ }^{9}$ Department of Medical Genetics, Yorkhill Hospitals, Yorkhill, Glasgow, UK; ${ }^{10}$ Department of Child Health, Royal Hospital for Sick Children, Glasgow, UK; ${ }^{11}$ Département de Génétique, Université Paris Descartes, Paris, France; ${ }^{12}$ Department of Clinical Genetics, Royal Devon and Exeter Hospital, Exeter, UK; ${ }^{13}$ Department of Human Genetics, Radboud University Nijmegen Medical Centre, Nijmegen, The Netherlands; ${ }^{14}$ Institutut für Humangenetik, Universitätsklinikum Essen, Essen, Germany

*Correspondence: Dr R Newbury-Ecob, Clinical Genetics Department, University Hospitals NHS Foundation Trust, St Michael's Hospital, Southwell Street, Bristol BS2 8EG, UK. Tel: +44 117928 5107; Fax: +44 117928 5108; E-mail: Ruth.Newbury-Ecob@UHBristol.nhs.uk

Received 22 September 2010; revised 18 November 2010; accepted 25 November 2010; published online 2 February 2011 
precursors were identified by monitoring specific ions and quantitation was achieved using an internal standard and calibration material with known amounts of each cholesterol. These studies were performed in patients 1,2 , and 3 .

\section{Genetic study: array comparative genomic hybridization}

Array comparative genomic hybridization (CGH) was performed using standardized protocols with differing platforms used according to local arrangements (using the Bluegnome cytochip $1 \mathrm{Mb}$ BAC array, tiling path $32 \mathrm{~K}$ BAC array, ${ }^{5}$ or the Agilent Wessex customized $44 \mathrm{~K}$ oligoarray). All patients except patients 4 and 12 had array CGH performed.

\section{RESULTS}

\section{Clinical data}

The clinical details of patients are described below and summarized in Table 1. Facial photographs of patients are shown in Figure 1 and general photos in Figure 2.

Patients 1 and 2 have previously been reported as they are the only siblings reported with clinical features of BOS. ${ }^{4}$ Patient 1 is a male born to non-consanguineous Caucasian British parents. He was born at 38 weeks gestation by emergency cesarean section for fetal distress. His birth weight was $3.23 \mathrm{~kg}$ (10th centile) and OFC $33.8 \mathrm{~cm}$ (25th centile). He had trigonocephaly, prominent eyes with upslanting palpebral fissures and hypertelorism, depressed nasal bridge, low-set and dysplastic ears, narrow U-shaped palate with broad alveolar ridges, micrognathia, sacral dimple, and nevi flammei on the philtrum, nape of the neck, and forehead. He had truncal hypotonia, but limb hypertonia with an unusual posture characterized by the shoulders being held externally rotated and adducted, elbows flexed, wrists flexed and ulnar deviated, and ulnar deviation at the metacarpophalangeal joints (referred to from here on as the 'BOS posture'), and also contractures of the elbows and fingers and gracile ribs. Initially he had respiratory distress and episodes of hypoglycemia. $\mathrm{He}$ had significant feeding difficulties, recurrent vomiting, and failure to thrive requiring gastrostomy feeding and fundoplication surgery. Feeding has improved, but he continues to be gastrostomy fed along with some oral intake. At age 14 he had bowel obstruction secondary to volvulus requiring partial colectomy. In early childhood he had significant problems with recurrent chest and urinary tract infections, which have become less frequent. He has a unilateral squint. Weight and head circumference rapidly dropped in infancy to below the 0.4 th centiles, but have stabilized from early childhood, and at age 15 years his height is $123 \mathrm{~cm}(\ll 0.4$ th centile), weight $27.0 \mathrm{~kg}(<0.4$ th centile), and OFC $49.5 \mathrm{~cm}(\ll 0.4$ th centile). He has severe developmental delay, but has made slow developmental progress, being able to stand with support and showing reasonable understanding, but without meaningful communication. Cranial MRI showed a relatively small brain with expansion of right frontal horn of the lateral ventricle, slender corpus callosum and brainstem, and abnormal neuronal migration with focal subependymal nodular heterotopia.

Patient 2 was born at 37 weeks gestation by emergency cesarean section owing to placenta previa. She weighed $2.56 \mathrm{~kg}$ (25th centile) and had a head circumference of $32.7 \mathrm{~cm}$ (25th centile). The pregnancy was complicated by gestational diabetes. Dysmorphic features were similar to her brother. She had the same 'BOS posture' with fixed contractures at the elbows. Clinical course and developmental progression has been similar, but slightly worse than her brother. In early childhood infections were a recurrent problem, particularly chest infections, and also pneumococcal meningitis. Problems with feeding and recurrent vomiting have required gastrostomy feeding and fundoplication, vomiting has improved but she remains gastrostomy fed.
Table 1 Clinical features of reported and previously reported patients with BOS

\begin{tabular}{lcc}
\hline & $\begin{array}{c}\text { Previously } \\
\text { reported }\end{array}$ & $\begin{array}{c}\text { Our } \\
\text { patients }\end{array}$ \\
\hline Gender & 7 Male: & 4 Male: \\
Death <2 years & 9 female & 10 female \\
& $8 / 16$ & $4 / 14$ \\
Clinical & & \\
Feeding difficulties/FTT & & \\
IUGR & $14 / 14$ & $14 / 14$ \\
Severe/profound LD & $14 / 16$ & $11 / 14$ \\
Recurrent infections & $10 / 10$ & $14 / 14$ \\
Seizures & $5 / 12$ & $11 / 14$ \\
Arrhythmias & $7 / 11$ & $6 / 14$ \\
Apneas & $4 / 11$ & $0 / 14$ \\
& $7 / 12$ & $4 / 14$
\end{tabular}

\section{Craniofacial}

Microcephaly

Trigonocephaly

Micro/retrognathia

Flammeus nevus

Prominent eyes

Abnormal palate

Hypertelorism

Upslanting palpebral fissures

Epicanthal folds

Broad alveolar ridges

Cleft/notch Lip

Cleft palate

Buccal frenulae

Depressed nasal bridge

Anteverted nares

Low-set posteriorly rotated ears

$\begin{array}{cc}9 \text { primary/ } & 4 \text { primary/10 } \\ 4 \text { secondary } & \text { secondary } \\ 16 & 12 \\ 12 & 13 \\ 16 & 12 \\ 14 & 14 \\ 15 & 10 \\ 8 & 9 \\ 13 & 7 \\ 1 & 5 \\ 8 & 11 \\ 9 & 2 \\ 7 \text { unilateral/ } & 2 \text { unilateral/ } \\ 3 \text { bilateral } & 1 \text { bilateral } \\ 4 & 0 \\ 16 & 7 \\ 10 & 8 \\ 14 & 8\end{array}$

Ophthalmic

Strabismus

Anterior chamber abnormalities

Myopia

Retinal/optic nerve abnormalities

Hair/skin

Low hairline

Hypertrichosis

8

7

Neurological/skeletal

BOS posture

Fixed contractures

Congenital dislocations

Hypotonia

Hypertonia

Brain abnormalities

Other

Genital abnormalities

Renal abnormalities

Cardiac abnormalities

$\begin{array}{rr}7 & 8 \\ 2 & 4 \\ 2 & 8 \\ 13 & 5\end{array}$

Abbreviations: BOS, Bohring-Opitz syndrome; FTT, failure to thrive; IUGR, intauterine growth restriction (birthweight $<10$ th centile); LD, learning difficulties. Bold-suggested diagnostic criteria. 

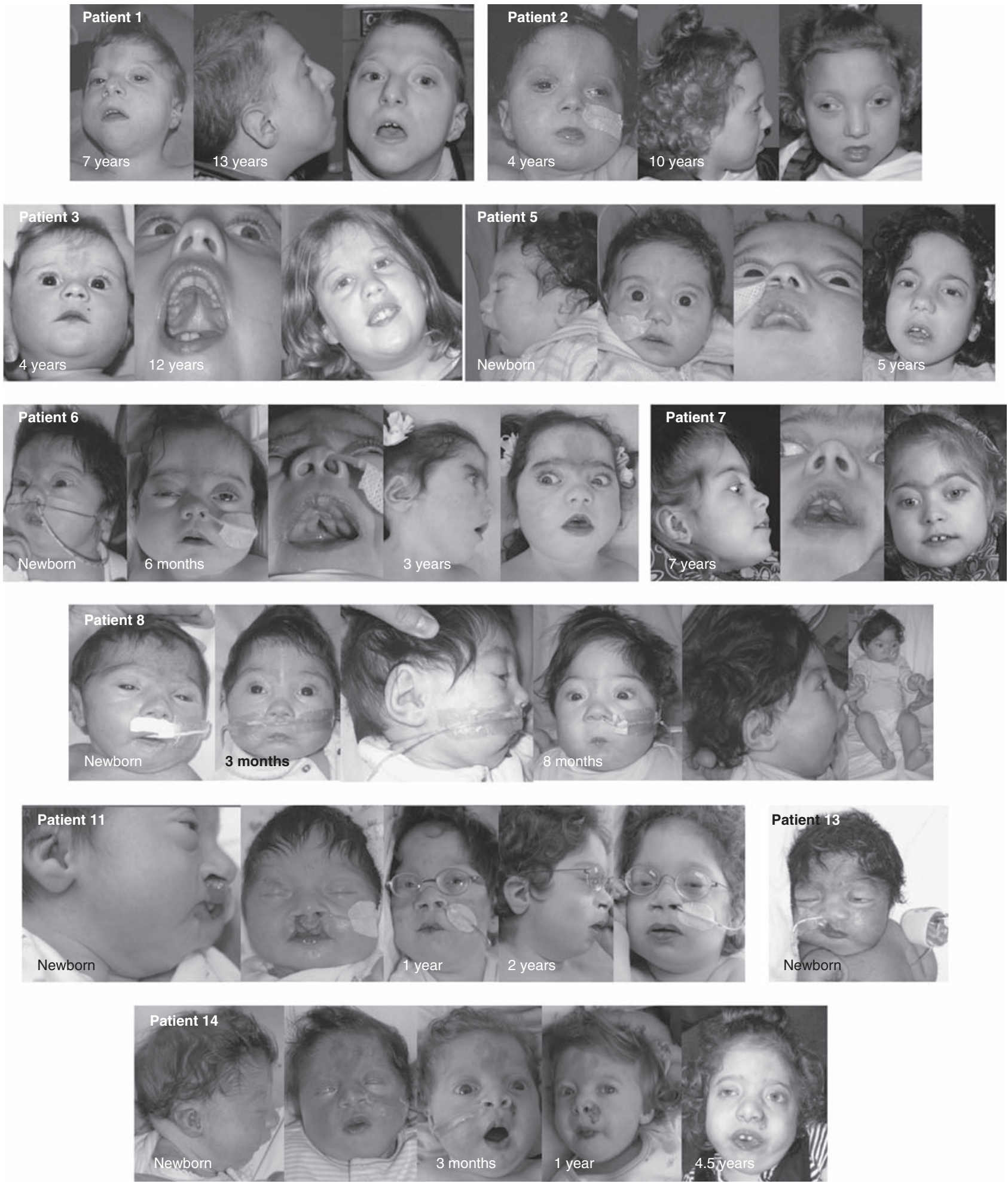

Figure 1 Facial appearance of features of patients with BOS.

She has a squint and had delayed visual maturation. Absence seizures in early childhood were treated with levitaracetam. She also had cyanotic/apneic episodes. At age 10 years she was diagnosed with proximal renal tubular acidosis leading to fragility fractures. Her growth has shown the same pattern as her brother and at 12 years of age her height is $117 \mathrm{~cm}(<0.4$ th centile), weight $25.2 \mathrm{~kg}$ (0.4th-2nd centile), and OFC $49.5 \mathrm{~cm}(\ll 0.4$ th $)$. At 12 years of age she can sit unsupported at times and babbles, but has no meaningful communication. Cranial MRI has shown a relatively small brain with expansion of the frontal horns of the lateral ventricles and nodular 


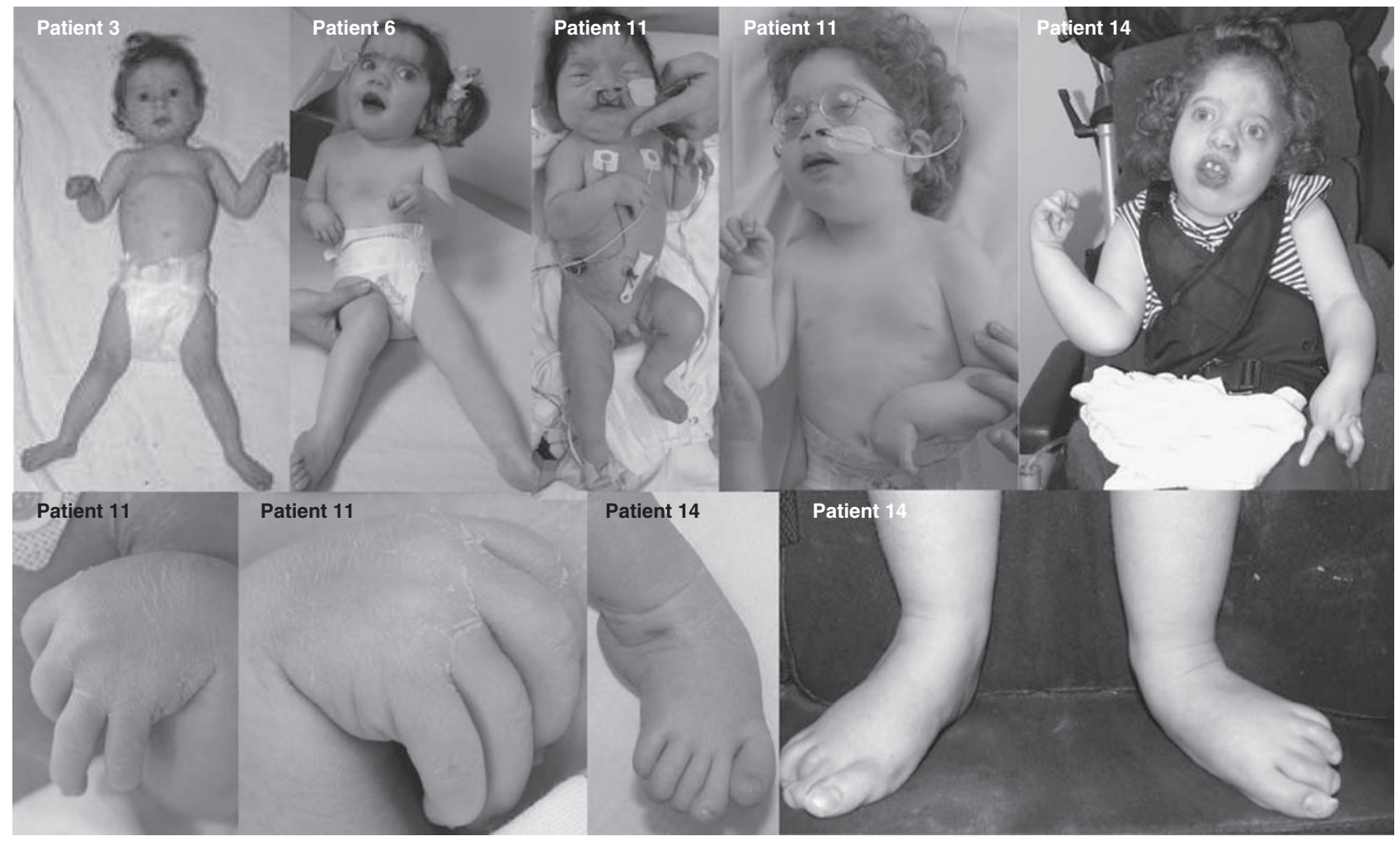

Figure 2 Pictures showing the typical posture of patients with BOS.

heterotopic gray matter in the wall of the left lateral ventricle. Echocardiogram showed a mild pulmonary artery stenosis and patent foramen ovale.

Patient 3 was born at 41 weeks gestation to non-consanguineous Caucasian British parents with a birth weight of $3.0 \mathrm{~kg}$ (10th centile). She had trigonocephaly, glabella nevus flammeus along with multiple capillary hemangiomas on the trunk, prominent eyes with hypoplastic supraorbital ridges, low-set ears with overfolded helices, broad alveolar ridges with a narrow $\mathrm{V}$-shaped palate and abnormal uvula, deep transverse palmar creases, and long overlapping fingers and toes with partial $2 / 3$ syndactyly. In recent years, the eyes and trigonocephaly have become less prominent and the flammeus nevus has faded. She has developed small, puffy, cold feet, fetal finger pads have been noted, and she has slowly progressive scoliosis. She was hypotonic and had BOS posture along with congenital hip dislocation, radial head dislocation, and mild pectus excavatum. In infancy she had absence seizures and apneic episodes. Problems with recurrent chest infections in infancy and early childhood have since improved. Other problems included constipation, absent lacrimation, hyperhidrosis, and persistent primary dentition. She has unilateral squint, ptosis, and high myopia. Poor feeding requiring gastrostomy feeding and gastroesophageal reflux were problematic in early childhood, but have since almost resolved. Indeed she is now overweight, and at the age of 14 years she weighs $54 \mathrm{~kg}$ (50th centile), having been below the 0.4 th centile, with height $141 \mathrm{~cm}(<0.4$ th centile) and OFC $50.5 \mathrm{~cm}$ ( $\ll 0.4$ th centile). Development was severely delayed, but has made rapid progress in the past few years. She has been able to walk a few steps since the age of 7 , and despite no speech at 10 years of age, she now has a good vocabulary and can form simple sentences. Recent problems with snoring and sleep apnea have been improved with adenotonsillectomy. At 13 years of age she was diagnosed as an insulin-dependent diabetic (although she has a strong family history of this).

Patient 4 was born at term to non-consanguineous Afro-Caribbean parents. Birth weight was $2.1 \mathrm{~kg}(<0.4$ th centile) with a length of $47 \mathrm{~cm}$ (2nd-9th centile) and OFC of $34 \mathrm{~cm}$ (25-50th centile). She had prominent eyes with hypoplastic supraorbital ridges, hypertelorism, upslanting palpebral fissures, epicanthal folds, glabella nevus flammeus, depressed nasal bridge with anteverted nares, low-set ears, retrognathia, low temporal hairline, synophrys, hypertrichosis and deep palmar creases. BOS posture was present. Feeding was a significant problem and required gastrostomy feeding. She had high myopia. Secondary microcephaly developed, and at the age of 2 years and 10 months her height was $73.7 \mathrm{~cm}$ (0.4th centile) and weight $8.7 \mathrm{~kg}(<0.4$ th centile). Echocardiogram showed a patent ductus arteriosus and cranial MRI showed a Dandy-Walker malformation. She died at 4 years and 7 months of age owing to pneumonia.

Patient 5 was born at 41 weeks gestation by cesarean section for transverse lie to non-consanguineous Orthodox (Ashkenazi) Jewish British parents. She is the 11 th of 12 children with no similar problems noted in any other family members. Birth parameters were atypical with weight $3.83 \mathrm{~kg}$ (75th centile) and OFC $35.8 \mathrm{~cm}$ (75th centile). She had prominent eyes with hypoplastic supraorbital ridges, glabella nevus flammeus, depressed nasal bridge and anteverted nares, lowset posteriorly rotated ears, small mouth, broad alveolar ridges with a high U-shaped narrow palate, submucous cleft, and choanal atresia, low temporal hairline, retrognathia, BOS posture, and bilateral knee dislocations and unilateral hip dislocation. She is gastrostomy fed owing to feeding difficulties. Duodenal atresia was operated on at day 3 of life. At the age of 5 years she takes small amounts of food orally, but remains predominantly gastrostomy fed. Ophthalmic problems include high myopia and persistent pupillary membrane. At the age of 
5 years her height is $99 \mathrm{~cm}$ ( 0.4 th centile), weight $16 \mathrm{~kg}$ (2nd centile), having dropped below the 0.4 th centile in infancy, and OFC $47 \mathrm{~cm}$ $(<0.4$ th centile). Development has been severely delayed, she has been able to stand from the age of 3 years, and at 5 years is starting to communicate through picture signs and pointing. Echocardiogram showed mild pulmonary stenosis, ASD, and septal hypertrophy causing dynamic left ventricular outflow obstruction, which resolved by 6 months of age.

Patient 6 was born at term to non-consanguineous Caucasian British parents weighing $2.3 \mathrm{~kg}$ ( 0.4 th centile). She had trigonocephaly, prominent eyes with hypoplastic supraorbital ridges, glabella nevus flammeus, broad alveolar ridges, low-set dysplastic ears, retrognathia, low temporal hairline, and hypertrichosis. In infancy she was hypotonic and displayed the BOS posture, along with having flexion contractures at wrists and elbows, bilateral radial head dislocation and bilateral congenital hip dislocation, overlapping fingers, partial $2 / 3$ syndactyly, and a mild pectus excavatum. She has had severe feeding problems along with failure to thrive requiring gastrostomy feeding, but no evidence of significant reflux. In infancy she had 'choking' episodes of uncertain cause and recurrent chest infections, which improved by early childhood. Development is severely delayed, but from 18 months of age she has developed social behaviors. She has significant myopia and reduced hearing on the right side. At 2.5 years of age her height is $73 \mathrm{~cm}(\ll 0.4$ th centile $)$, weight $6.65 \mathrm{~kg}(\ll 0.4$ th centile), and OFC $43.6 \mathrm{~cm}$ ( $\ll 0.4$ th centile). Imaging has shown dextrocardia and cerebellar vermis hypoplasia, Dandy-Walker malformation, and thin corpus callosum.

Patient 7 has previously been reported as part of a series discussing determination of pathogenicity in inherited cytogenetic abnormalities. ${ }^{5}$ She was born at 35 weeks gestation to non-consanguineous Caucasian Danish parents with a birth weight of $1.82 \mathrm{~kg}$ (10th) and OFC $28.3 \mathrm{~cm}$ (0.4th centile). She had trigonocephaly with a fused metopic suture (requiring surgery at 9 months of age), prominent eyes, upslanting palpebral fissures, epicanthus, glabella nevus flammeus, high and narrow palate, hypertrichosis, low temporal hairline, synophrys, retrognathia, deep palmar creases, and sacral dimple. BOS posture and hypotonia were noted, with contractures at hips, knees, and ankles, and development of a scoliosis. She had feeding difficulties and failure to thrive requiring gastrostomy feeding. Development was severely delayed with no development of speech or ability to sit. Recurrent chest infections and difficult to manage generalized seizures became apparent in infancy. Ophthalmic problems included a squint and poor vision with myopia. At the age of 7 years her height is $98 \mathrm{~cm}$ $(<0.4$ th centile), weight $15.3 \mathrm{~kg}$ ( 0.4 th centile), and $\mathrm{OFC} 44.5 \mathrm{~cm}$ ( $\ll 0.4$ th centile). Cranial MRI showed generalized atrophy.

Patient 8 was born at term to non-consanguineous Caucasian French parents. At birth she weighed $2.2 \mathrm{~kg}$ (0.4th centile), length was $43 \mathrm{~cm}(<0.4$ th centile), and OFC $31 \mathrm{~cm}$ (0.4th centile). She had trigonocephaly, glabella nevus flammeus, prominent eyes with hypoplastic supraorbital ridges, hypertelorism, epicanthal folds, broad alveolar ridges, low temporal hairline, hypertrichosis, and retrognathia. BOS posture was present along with long overlapping fingers. She had feeding difficulties and failure to thrive requiring gastrostomy feeding. Ophthalmic problems included squint and ptosis. At 10 months her height was $70 \mathrm{~cm}$ (25th centile), weight $8 \mathrm{~kg}$ (9th-25th centile), and OFC $42 \mathrm{~cm}$ ( $<0.4$ th centile). Echocardiogram showed pulmonary valvular stenosis and cranial MRI showed enlarged lateral ventricles. Bone age was advanced. She died at 11 months of age.

Patient 9 was born at 37 weeks gestation by cesarean section owing to reduced fetal movements to non-consanguineous parents from Morocco. Birth weight was $2.4 \mathrm{~kg}$ (9th centile), length $46 \mathrm{~cm}$ (9th centile), and OFC $32 \mathrm{~cm}$ (9-25th centile). Dysmorphic features included trigonocephaly with fused metopic suture, prominent eyes, hypertelorism, epicanthal folds, glabella nevus flammeus, depressed nasal bridge, broad alveolar ridges, retrognathia, and low temporal hairline. BOS posture was present along with contractures of fingers, knees, and elbows with limited spontaneous movement. Development was severely delayed. She had feeding difficulties and failure to thrive. She has had generalized seizures and EEG showed hypsarrhythmia. Ophthalmic problems consisted of squint and nystagmus. She also had an umbilical hernia. At 15 months of age her height was $66 \mathrm{~cm}$ $(<0.4$ th centile), weight $7.9 \mathrm{~kg}(<0.4$ th centile) and OFC $36 \mathrm{~cm}$ $(\ll 0.4$ th centile). Cranial MRI showed leukomalacia. Recurrent infections were problematic and she died at 2 years of age owing to pneumonia.

Patient 10 was born at term to non-consanguineous Turkish parents. Birth weight was $1.8 \mathrm{~kg}(<0.4$ th centile) and length $42 \mathrm{~cm}$ $(<0.4$ th centile). Dysmorphic features included prominent eyes, upslanting palpebral fissures, broad alveolar ridges, retrognathia, low temporal hairline, and hypertrichosis. BOS posture was present, the thumbs were adducted, and the fingers had contractures as well as elbows and knees. Scoliosis was also present. She had median cleft palate, feeding difficulties requiring tube feeding, and failure to thrive. Other problems included recurrent infections and profound sensorineural hearing loss. Ophthalmological examination revealed atrophy of retinal epithelium and nystagmus. Seizures developed in infancy and were treated with valproate. Growth parameters fell further from the normal range, and at the age of 5 years she had a height of $90 \mathrm{~cm}(<0.4$ th centile), a weight of $12 \mathrm{~kg}$ ( $<0.4$ th centile), and an OFC $44 \mathrm{~cm}(\ll 0.4$ th centile). Echocardiography showed a secundum atrial septal defect. She died at the age of 6 years owing to pneumonia.

Patient 11 was born by cesarean section at 38 weeks of gestation to non-consanguineous Dutch Caucasian parents with a birth weight of $2.97 \mathrm{~kg}$ (25th centile). Bilateral cleft lip and clenched hands had been detected antenatally. He had bilateral cleft lip and palate, prominent eyes with hypoplastic supraorbital ridges, hypertelorism, midfacial capillary hemangioma, thick hair with low temporal hairline, and absence of distal interphalangeal creases from the 2nd-4th fingers of the right hand and 3rd and 4th fingers of the left hand. He had feeding difficulties and failure to thrive. Inguinal hernia was corrected at 2.5 months of age. He had high myopia, squint, and high intraocular pressures initially. BOS posture, hypotonia, and bilateral hip dysplasia were present. At 8 months of age, absence seizures developed with tonic-clonic seizures appearing at 1 year of age, with epileptiform activity being confirmed on EEG. Head circumference was $43 \mathrm{~cm}$ at 9.5 months of age (0.4th centile) and trigonocephaly became evident at 1 year of age. Development has been severely delayed, and at 2.5 years of age he can roll over but cannot sit unsupported and does not speak. His height is $98 \mathrm{~cm}$ (98th centile) and weight $14 \mathrm{~kg}$ (50th centile). Cranial MRI showed partial agenesis of the corpus callosum.

Patient 12 was born at term to consanguineous Pakistani parents weighing $2.16 \mathrm{~kg}$ (2nd centile). He had feeding difficulties and failure to thrive. He had low temporal hairline, synophrys, facial hemangioma, prominent eyes with hypoplastic supraorbital ridges, hypertelorism, short philtrum, tented upper lip, high V-shaped palate with thickened alveolar ridges, micrognathia, hyperconvex nails, BOS posture, and rocker-bottom feet. He also had bilateral hypoplastic irides with anterior segment dysgenesis, scleralization of superior cornea, markedly cupped pale discs, pale retinal pigment epithelium/ prominent choroidal vessels, salt and pepper retinal changes, poorly defined macular regions, and high myopia. He developed secondary 
microcephaly. Cranial MRI showed agenesis of the corpus callosum and cerebellar hypoplasia. Echocardiogram showed hypertrophic cardiomyopathy. He died at 13 months from sepsis.

Patient 13 was born at 38 weeks gestation by elective cesarean section to non-consanguineous Indian parents. Antenatally a nuchal fold measurement of $2.4 \mathrm{~mm}$ (normal $<2 \mathrm{~mm}$ ) and IUGR were noted. He was born weighing $2.3 \mathrm{~kg}$ (2nd-9th centile) and OFC of $33 \mathrm{~cm}$ (25th centile). He had a small anterior fontanelle, ridged metopic suture, unilateral cleft lip, broad alveolar ridges, high palate, long uvula, prominent eyes with proptosis and hypertelorism, persistent pupillary membranes, low-set dysplastic ears, micrognathia, short neck, wide spaced nipples, hypotonia, and BOS posture with long clenched fingers and right positional foot deformity, but no true contractures initially. At 2 months of age, knee and elbow flexion deformities were more prominent with contractures of the elbows developing. Inguinal hernia was also noted. Feeding difficulties and reflux required nasogastric feeding. Echocardiogram showed a PFO. CT head was normal. Hearing assessment showed no response on the left and normal on the right. He had recurrent chest infections and died of cardio-respiratory problems, preceded by apneic episodes and bradycardias at 6 months of age.

Patient 14 has previously been reported to highlight the ophthalmic features of BOS, particularly high myopia. ${ }^{6}$ She was born at term to non-consanguineous Caucasian British parents weighing $2.9 \mathrm{~kg}$ (10th centile) and OFC was $32 \mathrm{~cm}$ (2nd centile). Antenatally a raised nuchal translucency was noted. She had trigonocephaly, facial flammeus nevus, a capillary hemangioma on the left cheek, low anterior hairline, upslanting palpebral fissures and prominent eyes, depressed nasal bridge and anteverted nares, small mouth, broad alveolar ridges and a narrow high-arched palate with sub-mucous cleft, along with the BOS posture and other joint abnormalities (genu recurvatum, congenital hip dysplasia, and knee and ankle dislocations). She required gastrostomy feeding for feeding difficulties and failure to thrive. She has had frequent respiratory infections and an episode of pneumococcal meningitis. She has had difficulties with upper airway obstruction and has required grommets for moderate conductive hearing loss. In infancy she had focal and generalized seizures, but these are well controlled with valproate. She had bilateral femoral fractures at the age of 2 years owing to osteopenia of unknown cause. She has exophthalmos, strabismus, retinal abnormalities, and high myopia. Development has been profoundly delayed, and at the age of 4.5 years she is unable to sit independently or make any meaningful communication. Her OFC is $46.5 \mathrm{~cm}(\ll 0.4$ th centile).

\section{Analysis of the cholesterol biosynthesis pathway}

Compared with normal plasma samples, there was no increase in the sterol profile in patients 1, 2 and 3 (other patients were not analyzed in this manner). 7 -DHC was analyzed in patient 9 and was normal.

\section{Array CGH}

Patient 7 had a maternally inherited deletion on chromosome 10q11.21-23 detected by metaphase high-resolution CGH. This was also found in two healthy siblings, and was similar to deletions previously reported without a recognizable phenotype, and was therefore felt unlikely to be pathogenic. Patient 10 had a maternally inherited 388-kb deletion at 13q33.3 not containing any known OMIM Morbid genes, and again therefore was felt unlikely to be pathogenic.

\section{DISCUSSION}

From the published patients and our cohort, the consistent clinical features of this rare condition are becoming clearer.

\section{Craniofacial features}

Key diagnostic features are microcephaly and trigonocephaly (Figure 1). Trigonocephaly is usually due to prominence of the metopic ridge and bitemporal narrowing, with few having true metopic synostosis. It may not be apparent in the neonatal period and typically becomes less evident through early childhood. Microcephaly may be either primary or secondary, in either case tending to become increasingly pronounced through early childhood. Palatal abnormalities are a key feature. Sometimes cleft palate, or submucous cleft, is present, but typically the palate is just abnormal with a high narrow grooved palate. Prominent eyes and hypoplastic supraorbital ridges, upslanting palpebral fissures, depressed nasal bridge and anteverted nares, facial flammeus nevus (which becomes less obvious with age), and low-set posteriorly rotated ears are almost universal. A low anterior/temporal hairline is seen in most patients in the neonatal period, but may become less pronounced with age. The older children show variability in their facial appearance. Some retain their appearance in infancy, but with some features such as trigonocephaly, eye prominence, and micrognathia becoming less prominent (eg, patients 1, 2, 3, and 5). In others, hypertrichosis and synophrys become more prominent and coarseness of the facial features can develop (eg, patients 6, 7, and 14). Hypertelorism, epicanthal folds, small mouth, broad alveolar ridges, and buccal frenulae (not seen in our series but reported previously) are found in some.

\section{Ophthalmic features}

Strabismus and retinal abnormalities are common (50\%), as are anterior chamber abnormalities (20\%) and high myopia (33\%).

\section{Growth and development}

IUGR is seen in most (83\%) and all who survive have feeding difficulties and fail to thrive. This is a key feature of BOS with gastrostomy feeding required from early infancy in all the patients we are aware of. In some this diminishes as a problem through childhood such that gastrostomy feeding may not be required in later childhood. Even in those who take food orally, they are often reported as having relatively little interest in food and poor coordination of swallowing. Despite this, during childhood weight increases relative to height and is associated with central obesity in some, also shown in the patient reported by Pierron et al. ${ }^{8}$

\section{Neurological and musculoskeletal features}

Structural brain abnormalities are commonly identified (70\%) but nonspecific. Reported findings include ventriculomegaly, delayed myelination, Dandy-Walker malformation, generalized atrophy, slender corpus callosum and brainstem, and neuronal migration abnormalities. One of the key features of BOS, which has been reported but not highlighted previously, is the unusual and characteristic limb posture these children adopt (Figure 2). This 'BOS posture' is recognized in all patients, although is not entirely specific for BOS. It appears unrelated to overall tone, structural brain abnormalities, or joint contractures/dislocations. Older patients may have purposeful movement of the limbs, but return to this posture as their resting state. Contractures are common (60\%) and congenital dislocations are seen in many if investigated for (33\%), with hip and radial head dislocations being the most common. In the older children, joint problems remain problematic and may prove to be a significant because of morbidity in these patients through adulthood. A few of our cohort have developed significant scoliosis, although at present none of these have required surgery. Two patients have had femoral fragility 
fractures, in one patient attributed to renal tubular acidosis and the other with osteopenia of unknown cause.

\section{Internal organ abnormalities}

Cardiac abnormalities are seen in almost half, but are nonspecific. Atrial septal defects, patent ductus arteriosus, and valvular abnormalities (most commonly pulmonary stenosis) have been reported. Septal hypertrophy was seen in two patients, but, despite being severe enough to cause obstruction in the neonatal period, improved in both patients. Intra-abdominal organs are rarely noted to be abnormal. Bowel malrotation and inguinal hernias have been reported in some.

\section{Morbidity and outcome}

There remains a high rate of infant mortality in this condition (40\%), most commonly owing to infections. If the early childhood period is survived, then many of the common problems, such as feeding difficulties and recurrent infections, become less problematic. Severe to profound developmental delay is universal, although there is some variability in terms of the level of communication and mobility achieved. One of the patients has made significant developmental progress in later childhood despite limited progress before this. Central obesity, sleep apnea, and type 1 diabetes have been noted in adolescence, but numbers are too small to generalize whether these are more common in BOS.

The cause and inheritance of BOS are unknown. Apart from our published report of siblings with BOS, ${ }^{4}$ patients 1 and 2 in this report, all cases have been sporadic. One patient came from a consanguineous family lending support to the likelihood of recessive inheritance. However, some of the families without recurrence are large, for example, patient 6 has 11 unaffected siblings. Presuming a genetic etiology, it therefore remains unclear as to whether BOS is caused by dominant mutations, with a germline mosaicism risk, or is a recessive disorder. It is also possible that there may be separate disorders with closely overlapping phenotypes but differing etiologies.

Patients have been identified with phenotypes resembling BOS owing to chromosome abnormalities, the most similar being a patient with trisomy 3 pter. $^{9}$ Mutations in the TACTILE gene have been implicated as a cause of Opitz C syndrome, which has overlapping features with BOS, after one patient was found to have a balanced translocation causing disruption of this gene ${ }^{10}$ and a missense mutation was subsequently identified in another. ${ }^{11}$ However, the clinical phenotype of these two patients is not typical of BOS and mutations of this gene have been excluded in patients with BOS, including several of those described here.

This report helps to delineate the specific clinical phenotype of BOS, and provide insight into the frequency and spectrum of clinical problems encountered and their outcome. Identifying the cause of BOS is essential to clarify whether this clinical phenotype is a single entity, and indeed whether more varied phenotypes are owing to the same cause but without the characteristic features. With the advent of next-generation sequencing technologies, it is anticipated that the basis of BOS, and other rare disorders of unknown etiology, will become apparent in the near future and lead to an improvement in our understanding of the molecular processes underpinning them.

\section{CONFLICT OF INTEREST}

The authors declare no conflict of interest.

\section{ACKNOWLEDGEMENTS}

We thank all of the patients and their families and Dr Kaname for analysis of the TACTILE gene in several patients.

1 Bohring A, Silengo M, Lerone M et al: Severe end of Opitz trigonocephaly (C) syndrome or new syndrome? Am J Med Genet 1999; 85: 438-446.

2 Oberklaid F, Danks DM: The Opitz trigonocephaly syndrome. Am J Dis Child 1975; 129: $1348-1349$.

3 Addor MC, Stefanutti D, Farron F et al: ' $C$ ' trigonocephaly syndrome with diaphragmatic hernia. Genet Counsel 1995; 6: 113-120.

4 Greenhalgh KL, Newbury-Ecob RA, Lunt PW, Dolling CL, Hargreaves H, Smithson SF: Siblings with Bohring-Opitz syndrome. Clin Dysmo 2003; 12: 15-19.

5 Bisgaard A-M, Kirchoff M, Nielsen JE et al: Transmitted cytogenetic abnormalities in patients with mental retardation: pathogenic or normal variants. Eur J Med Genet 2007; 50: 243-255.

6 Simpson AR, Gibbon CE, Quinn AG, Turnpenny PD: Infantile high myopia in BohringOpitz syndrome. J AAPOS 2007; 11: 524-525.

7 Kelley R: Diagnosis of Smith-Lemli-Optiz syndrome by gas chromatography/mass spectrometry of 7-dehydrocholesterol in plasma, amniotic fluid and cultured skin fibroblast. Clin Chim Acta 1995; 236: 45-58.

8 Pierron S, Richelme C, Triolo $\mathrm{V}$ et al: Evolution of a patient with Bohring-Opitz syndrome. Am J Med Genet 2009; 149A: 1754-1757.

9 McGaughran J, Aftimos S, Oei P: Trisomy of 3pter in a patient with apparent C (trigoncephaly) syndrome. Am J Med Genet 2000; 94: 311-315.

10 Chinen Y, Kaname T, Yanagi K, Saito N, Naritomi K, Ohta T: Opitz trigonocephaly C syndrome in a boy with a de novo balanced reciprocal translocation t(3;18)(q13.13;q12.1). Am J Med Genet 2006; 140A: 1655-1657.

11 Kaname T, Yanagi K, Chinen Y et al: Mutations in CD96, a member of the immunoglobulin superfamily, cause a form of the C (Opitz trigonocephaly) syndrome. Am J Hum Genet 2007; 81: 835-841. 